University of Nebraska - Lincoln

DigitalCommons@University of Nebraska - Lincoln

USDA National Wildlife Research Center - Staff Publications
U.S. Department of Agriculture: Animal and Plant Health Inspection Service

March 2001

\title{
Comparison of Primary and Secondary Repellents for Aversive Conditioning of European Starlings
}

Roger W. Sayre

APHIS/WS/National Wildlife Research Center, U.S. Department of Agriculture

Larry Clark

USDA/APHIS/WS National Wildlife Research Center, larry.clark@aphis.usda.gov

Follow this and additional works at: https://digitalcommons.unl.edu/icwdm_usdanwrc

Part of the Environmental Sciences Commons

Sayre, Roger W. and Clark, Larry, "Comparison of Primary and Secondary Repellents for Aversive Conditioning of European Starlings" (2001). USDA National Wildlife Research Center - Staff Publications. 611.

https://digitalcommons.unl.edu/icwdm_usdanwrc/611

This Article is brought to you for free and open access by the U.S. Department of Agriculture: Animal and Plant Health Inspection Service at DigitalCommons@University of Nebraska - Lincoln. It has been accepted for inclusion in USDA National Wildlife Research Center - Staff Publications by an authorized administrator of DigitalCommons@University of Nebraska - Lincoln. 
Published in Pesticides and Wildlife, edited by John J. Johnston. ACS Symposium Series 771. American Chemical Society, Washington, DC, 2001. 


\section{Chapter 23}

\section{Comparison of Primary and Secondary Repellents for Aversive Conditioning of European Starlings}

\author{
Roger W. Sayre and Larry Clark
}

\section{APHIS/WS/National Wildlife Research Center, U.S. Department of Agriculture, 4101 Laporte Avenue, Fort Collins, CO 80521-2154}

Secondary repellents have been demonstrated to effectively reduce avian crop depredation. However, these compounds frequently are toxins and there are concerns about environmental safety. Many primary repellents are toxicologically and environmentally safer, but these chemicals are not as effective when traditional delivery methods are used. We set out to determine whether the difference in efficacy of primary vs. secondary repellents was due to differences in potency of the chemical classes or site of action within the animal. We hypothesized that the efficacy of primary repellents could be enhanced if they could be delivered directly to the gastrointestinal system, thus by-passing the peripheral senses. Our experiments were conducted on captive European starlings (Sturnus vulgaris). We showed that by-pass of the peripheral senses via oral gavage enhanced the efficacy of 2

hydroxyacetophenone (2HAP), a primary repellent. However, we found that starlings were sensitive to changes in their visual environment, and that learned avoidance to a colored food cup could be mitigated by changing the cage door of the test system. Topical application of repellents to foods allowed starlings to selfmediate their exposure to the repellents. We found that methiocarb and methyl anthranilate (MA), a primary repellent, both induced food aversion learning, but 2HAP did not. Detailed analyses of feeding behavior elucidated the mechanistic differences between Methiocarb, MA, and 2HAP. Methiocarb induced gastrointestinal illness in starlings, which resulted in post-ingestive suppression of food intake, and subsequent food aversion learning. Some starlings with MA in their food also developed gastrointestinal illness, and food aversion learning. Starlings with $2 \mathrm{HAP}$ in their feed did exhibit reduced feeding efficiency, but these starlings did not develop gastrointestinal illness or food aversion learning. These experiments demonstrate that the location and intensity of illness are of paramount importance to induce food aversion learning.

\section{Introduction}

Chemical repellents are important management tools for reducing avian crop damage. Chemical repellents are categorized as either primary or secondary, and these categories operate by different mechanisms ${ }^{1}$. With secondary repellents, animals learn to avoid foods because they associate an aversive experience caused by the chemical (the unconditional stimulus), with paired ancillary sensory cues such as visual, olfactory, taste, or auditory stimuli (the conditional stimulus). Secondary repellents have been demonstrated to be effective at reducing crop depredation, and they have the advantage of promoting long-term learned avoidance. However, there are concerns about environmental and toxicological safety, which has made use of these chemicals less desirable $e^{2,3}$.

In contrast, primary repellents are immediately noxious to an animal and avoidance behavior is reflexive; i.e., learning is not required. In most cases primary repellents appear to cause irritation ${ }^{4}$. Chemically sensitive nociceptors of the trigeminal nerve detect chemicals and the animal perceives this neuronal activity as a sensation of pain. The reflexive avoidance response results in a limited exposure or dosage. Thus, the animal self-limits its exposure to the chemical and thereby limits the magnitude of the unpleasant experience. Because the strength of aversive conditioning is directly related to the intensity of the punishment ${ }^{5}$, primary repellents are less likely to promote strong conditioned avoidance. That is to say, primary repellents by their nature are infrequently consumed in sufficient quantity, in a short period of time, to be useful as effective agents in promoting strong learned avoidance. Rather, birds frequently sample foods containing primary repellents. Insufficient quantities of the repellents are consumed to promote learned avoidance. Consequently birds will frequently return to feed once these chemicals are removed ${ }^{6,7,8}$.

In spite of the limitations, there has been increased interest in developing primary repellents, especially those that are considered to be less toxic and environmentally harmful than secondary repellents ${ }^{9}, 10$. The primary repellents of interest are derived from natural food additives, which are safe for human consumption. In contrast, most effective secondary repellents (e.g., methiocarb) are toxicants derived from pesticides. The primary repellent methyl anthranilate (MA) is a grape flavored food additive that it is an irritant to birds. The current body of knowledge indicates that MA should be toxicologically and environmentally safe when used as a bird repellent. MA is toxic only at very high concentrations, and it 
biodegrades rapidly. MA is approved by the U.S. Food and Drug Administration as a food flavor additive and is classified as Generally Recognized as Safe (GRAS). Although primary repellents are safe, they are not considered to be as effective as secondary repellents. A possible method to circumvent the limitation that primary repellents do not promote long-term learned avoidance would be to by-pass the peripheral senses so the primary repellent is delivered into the gastrointestinal system. This would result in several advantages. The animals would not selfmediate the dosage, and could be exposed to more repellent. Second, gastrointestinal illness due to a repellent would increase the saliency of the unconditional stimulus in relation to feeding behavior, resulting in stronger aversive conditioning. Our objective was to by-pass the peripheral nociceptors and directly compare the illness promoting potential of primary and secondary repellents when delivered enterically. We tested 2 hydroxyacetophenone (2HAP) and MA, which were identified as primary repellents from structure-activity models and drinking trials $^{11,12,13}$. The secondary repellent, methiocarb, has been demonstrated to be an effective aversive conditioner ${ }^{6,14,15,16,17,18}$.

\section{Methods}

\section{Study Subjects}

During September 1998 we trapped European starlings $(n=40)$ in Fort Collins, Colorado. We conducted the 3 experiments from November 1998 to June 1999. Starlings were housed in visually isolated metal cages $(36 \mathrm{~cm} \times 23 \mathrm{~cm} \times 28$ $\mathrm{cm}$ ) (Animal Care Products, Bryan Texas). Temperature remained constant at $22^{\circ} \mathrm{C}$ during the study, and lighting was maintained on a seasonally shifting photo period. Each cage was provisioned with a food cup and a water bottle holder attached to the cage door, $5 \mathrm{~cm}$ above the wire mesh cage floor. We provided food (Purina Layena Checker) ad libitum in metal food cups ( $8.3 \mathrm{~cm}$ diameter), in addition to unlimited access to water. To establish baseline food intake, we measured food consumption of each starling for a period of 2 hours (commencing 2 hours after the onset of light) for 2 days prior to testing.

\section{Experiment 1}

The objective of this experiment was to by-pass the peripheral senses to compare the effect that primary and secondary repellents have on food avoidance learning in starlings. The primary repellent was 2 hydroxyacetophenone (2HAP), and the secondary repellent was methiocarb.

\section{Test Protocol}

We ranked starlings according to food intake and assigned them to 1 of 3 groups ( $n=8$ /group) so that each group had individuals with high, moderate, and low consumption rates. Treatments were randomly assigned to the groups. Treatments included control (no handling); gavage with methiocarb $(4 \mathrm{mg} / \mathrm{kg}$ ) diluted into propylene glycol (PG) at $2 \mathrm{ml} / \mathrm{kg}$; and $2 \mathrm{HAP}(250 \mathrm{mg} / \mathrm{kg})$, also diluted into PG (2 $\mathrm{ml} / \mathrm{kg}$ ). Dosages of methiocarb and PG followed Mason and coworkers ${ }^{15,16,19}$. We conducted preliminary range finding determine dosages of $2 \mathrm{HAP}$ and found that 250 $\mathrm{mg} / \mathrm{kg}$ was sufficient to cause temporary irritation and delay of food consumption (Sayre and Clark unpublished data).

The experiment was conducted during a period of 2 days for each bird. During the first day, i.e., training, we provided food in a colored cup (the conditional stimulus) for 2 hours. The cup was colored with orange and black vertical stripes, because previous reports have indicated that this was the most effective color pattern for a visual stimulus in conditioning trials ${ }^{15,16}$. We removed the food cup and immediately gavaged (orally intubated) the starlings with repellent (the unconditional stimulus). We measured food intake after gavage to evaluate the immediate effect of the repellent (the unconditional response). After 2 hours starlings were left undisturbed and were provided food ad libitum in uncolored cups. On the second day, 24 hours after exposure to the conditional stimulus, we conducted a two-choice feeding test, with food from an uncolored and a colored food cup, to determine if learned avoidance occurred (the conditional response).

\section{Training}

Approximately 16 hours prior to training we replaced the standard wire cage doors with plexiglass doors ( $0.64 \mathrm{~cm}$ thick). We installed these doors to enhance video taping and analysis for concurrent behavioral analyses, and we believed that 16 hours would be sufficient for the starlings to habituate. The placement of cup and water bottles on these doors was identical to the wire cage doors. On the training day, 2 hours after the onset of light, we replaced the standard metal food cup with a cup with orange and black vertical stripes (the conditional stimulus). Each cup contained $30 \mathrm{~g}$ of standard chow. After 2 hours of exposure to the conditional stimulus we removed the cup and immediately took the test starling from its cage and gavaged it with repellent solution. Control starlings were not handled. We held each starling for $1 \mathrm{~min}$ after gavage to prevent immediate regurgitation, and then returned the starling to its cage and presented it with $30 \mathrm{~g}$ of food in an uncolored food cup. We returned to measure food intake at 2,4 , and 6 hours post-gavage. Starlings were then left undisturbed until the following day.

\section{2-Choice Learning Test}

Two hours after the onset of light on the second day we conducted a 2choice learning test, with an uncolored metal cup and a colored cup with orange and 
black vertical stripes. Each cup contained $30 \mathrm{~g}$ of chow. The position of the cup (left or right) was determined randomly. After 2 hours, we measured food intake from each cup. We determined preference ratios by dividing consumption from the colored cup by consumption from both cups combined.

Analysis

We used a 2-factor repeated measures ANOVA to evaluate food intake on the day of training. The model included the independent variable for treatment with 3 levels (2HAP, methiocarb, and control). The dependent variable was the repeated measure of food intake at 2,4 , and 6 hours post-gavage. We used planned orthogonal contrasts to compare the average food intake by controls vs. $2 \mathrm{HAP}$ and methicarb (averaged during the 6 hour feeding period). In a separate analysis, to look for learning effects of treatment, we evaluated the data from the 2-choice learning test with a 1-way ANOVA. The preference ratios of controls vs. the treatment groups were compared with planned contrasts.

\section{Experiment 2}

The objective of Experiment 2 was to evaluate the effect of replacing the standard cage door with plexiglass. We tested starlings in 3 treatment groups ( $n=$ 6/group). Treated starlings were selected randomly among the starlings used in Experiment 1 . To minimize any effect of carryover learning, we waited $\geq 31$ days before re-testing any starling. Mason ${ }^{16,19}$ reported that learned avoidance lasted 1012 days following treatment with methiocarb, and we believe that the time between experiments was sufficient to minimize possible bias.

Treatments included oral gavage of PG $(2 \mathrm{ml} / \mathrm{kg})$ as control, $2 \mathrm{HAP}$ $(250 \mathrm{mg} / \mathrm{kg})$, and methiocarb $(4 \mathrm{mg} / \mathrm{kg})$. The repellents were diluted into PG (2 $\mathrm{ml} / \mathrm{kg}$ ). We used the same training and testing procedures as Experiment 1 with the following exceptions: (1) the standard metal cage doors remained on the cages and these were not replaced by the clear plexiglass doors; (2) we measured food consumption only during the first 2 hours post-gavage.

Analysis

We used a 1-way ANOVA and planned contrasts to compare food consumption of controls (PG) vs. $2 \mathrm{HAP}$ and methiocarb during the 2 hours immediately after gavage. The 2-choice preference ratios of starlings gavaged with PG, 2HAP, and methiocarb, measured on the following day, were also compared with a 1-way ANOVA and contrasts.

\section{Experiment 3}

The first objective of Experiment 3 was to evaluate the effect of increasing the duration of habituation to the plexiglass cage door from 16 hours to 60 hours. A second objective was to compare the behavioral responses of starlings to primary and secondary repellents when the peripheral senses are not bypassed.

\section{Adaptation}

To provide starlings a longer habituation period to the plexiglass cage door, we replaced the standard cage door with the clear plexiglass door 60 hours prior to training. In addition, we adapted the starlings to a schedule of mild food deprivation during the pre-training period. On the days prior to training (i.e., 48 and 24 hours before), we removed the food cup for 2 hours. After 2 hours we returned and provided the starlings with $30 \mathrm{~g}$ of standard chow for another 2 hours. We then measured food consumption to obtain a baseline food intake after mild food deprivation.

\section{Test Protocol}

We randomly assigned starlings to 1 of 4 groups, and randomly assigned treatments to the groups. Treatments included methiocarb $(0.2 \% \mathrm{w} / \mathrm{w})$, a secondary repellent; and primary repellents, $2 \mathrm{HAP}(1 \% \mathrm{w} / \mathrm{w})$ and methyl anthranilate (MA) $(0.8 \% \mathrm{w} / \mathrm{w})$. All repellents were diluted into ethyl alcohol, and the solution was spread topically onto the food 48 hours prior to feeding. The control was ethyl alcohol added to the food at $1 \% \mathrm{w} / \mathrm{w}$, which was the concentration we used with the repellent dilutions. After applying the repellent/ethyl alcohol mixture, we air dried the foods for $\mathbf{4 8}$ hours prior to feeding.

Like Experiments 1 and 2, Experiment 3 was conducted over 2 days with training and 2-choice learning phases. Two hours after sunrise on the training day, we removed each starlings's food cup for 2 hours. After 2 hours we provided food, according to preassigned treatment (the unconditional stimulus), in a colored cup (the conditional stimulus) for 2 hours. Each test starling was video-taped during the 2 hour training phase. We then removed the colored cup and weighed food consumption. Starlings were provided food ad libitum from a standard metal cup until the next day.

On the second day, 24 hours after exposure to treated food, we conducted a two-choice feeding test to determine if learning in response to the unconditional and conditional stimuli occurred. Two hours after a light onset we removed the food cup for two hours. We then provided standard chow in two food cups, an uncolored cup and a colored cup with orange and black vertical stripes, with each cup containing 30 $\mathrm{g}$ of standard chow. After 2 hours we measured food consumption from each cup and determined preference ratios by dividing consumption from the colored cup by consumption from both cups combined. 
Behavioral Analysis

We used an integrated video hardware and computer software package (Noldus Observer, Wanningen, The Netherlands) to quantify feeding behavior. During each $2 \mathrm{~h}$ observation period we measured the duration and frequency of feeding bouts; the frequency of eaten pellets, dropped pellets, failed attempts to eat, and drinks (Table 1). We calculated feeding efficiency as the number of pellets consumed per minute of feeding. To further evaluate the behavioral mechanisms in response to repellent-treated foods, we analyzed the frequency of feeding bouts, number of pellets eaten, and frequency of drinks at 30 minute intervals (i.e., 0 - 30 $\mathrm{min}, 30-60 \mathrm{~min}, 60-90 \mathrm{~min}$ and $90-120 \mathrm{~min}$ ). To evaluate the relationship between type of repellent and gastrointestinal illness we quantified the frequency of regurgitations during each $30 \mathrm{~min}$ interval.

\section{Table I. Categories and definitions for feeding and drinking behavior of} European starlings.

\begin{tabular}{|c|c|c|}
\hline $\begin{array}{l}\text { Category of } \\
\text { Behavior }\end{array}$ & $\begin{array}{l}\text { Measure of } \\
\text { Behavior }\end{array}$ & Definition \\
\hline Feeding bout & Duration \& Frequency & $\begin{array}{l}\text { Bird's bill is in food cup or bird } \\
\text { has food pellet in bill. }\end{array}$ \\
\hline Eat & Frequency & Bird consumes food pellet. \\
\hline Drop & Frequency & $\begin{array}{l}\text { Bird drops food pellet before } \\
\text { eating. }\end{array}$ \\
\hline Attempt & Frequency & $\begin{array}{l}\text { Bird searches in food cup but } \\
\text { does not hold food in bill. }\end{array}$ \\
\hline Drink & Frequency & Bird drinks from water tube. \\
\hline
\end{tabular}

Analysis

We used a 1-way ANOVA to evaluate the effect of repellent on food consumption during training. Food consumption of controls were compared against the repellent treatment groups with planned contrasts. We also used a 1-way ANOVA and planned contrasts to evaluate preference ratios of treated vs. controls. The feeding behavior data were analyzed by combining the primary repellents (2HAP and MA), which we compared with controls and methiocarb using planned contrasts.

\section{Results}

\section{Experiment 1}

The objective of Experiment 1 was to compare the effectiveness of gastrointestinal delivery of $2 \mathrm{HAP}$, a primary repellent, and methiocarb, a secondary repellent.

\section{Food Intake During Training}

Food intake on the day of training did not vary as a function of time and the type of treatment (Table II). However, starlings varied their food intake in response to the main effects of repellent treatment and time (Table II). Contrasts showed that relative to controls, starlings did not reduce their food intake after 2HAP gavage ( $P=$ 0.279 ) (Figure 1a). However, starlings treated with methiocarb reduced food consumption during the 2 hours following methiocarb gavage $(P=0.003)$

Table II. ANOVA for Food Consumption by starlings Following Gavage.

\begin{tabular}{llcl}
\hline Experiment & $d f$ & $F$ & $P$ \\
\hline Experiment 1 & 2,21 & 5.62 & 0.011 \\
$\quad$ Repellent & 2,21 & 69.86 & 0.001 \\
Time & 4,42 & 2.26 & 0.078 \\
Repellent $\times$ Time & & & \\
Experiment 2 & 2,15 & 5.67 & 0.015 \\
$\quad$ Repellent & & & \\
Experiment 3 & 3,20 & 2.01 & 0.14 \\
Repellent & & & \\
\hline
\end{tabular}

2-Choice Learning Test

Results from the 2-choice learning experiment indicated that the starlings did not develop a learned aversion to the visual cue $\left(\mathrm{F}_{2,21}=0.76 P=0.48\right)$, indicating that they failed to make the association between the physiological effects of the chemical used in the gavage and the visual stimulus (i.e., the vertically striped black and orange food cup). The contrasts showed that preference ratios of starlings treated with 2 HAP 
$(P=0.53)$ and methiocarb $(P=0.56)$ were similar to those observed in the control group (Figure 1b).
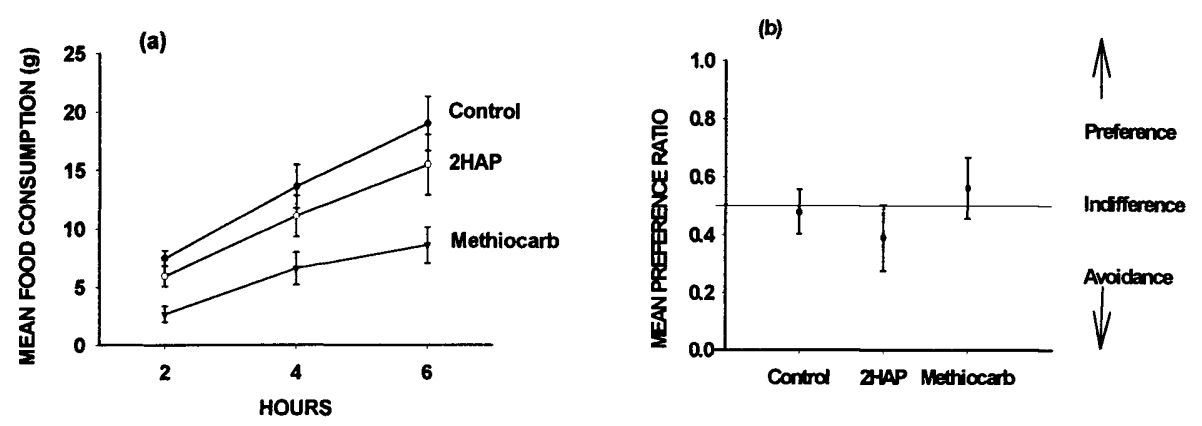

Figure la. Mean consumption (g) of food by starlings 2, 4, and 6 hours after gavage treatment ( $n=8 \mathrm{birds} /$ group). Figure $1 \mathrm{~b}$. Mean preference ratios $( \pm S E)$ of starlings during 2 choice test in Experiment 1.

\section{Experiment 2}

Experiment 2 also was conducted to compare the effect primary and secondary repellents had when the peripheral senses are bypassed. However, we first wanted to test the hypothesis that the starlings failed to develop an aversion to the conditional stimulus in Experiment 1 because they were distracted by the clear plexiglass door, and learning failed because of concurrent interference of the sign stimulus. That is to say the door was a more salient visual cue than the vertically striped color cup and, as a consequence, starlings failed to associate illness with feeding. We treated starlings with PG, 2HAP, and methiocarb but did not replace the standard wire cage door with plexiglass.

\section{Food Intake During Training}

The repellents affected food consumption (Table II). Relative to controls, food consumption by starlings was suppressed for starlings treated with $2 \mathrm{HAP}(P=$ $0.010)$ and methiocarb $(P=0.011)$ (Figure 2a).

\section{2-Choice Learning Test}

Under the conditions of the test we found that the starlings could be conditioned to avoid the colored food cup. Compared to controls, starlings treated with $2 \mathrm{HAP}(P=0.076)$ and methiocarb $(P=0.033)$ formed a conditioned avoidance to the visual cue (Figure $2 b$ ).
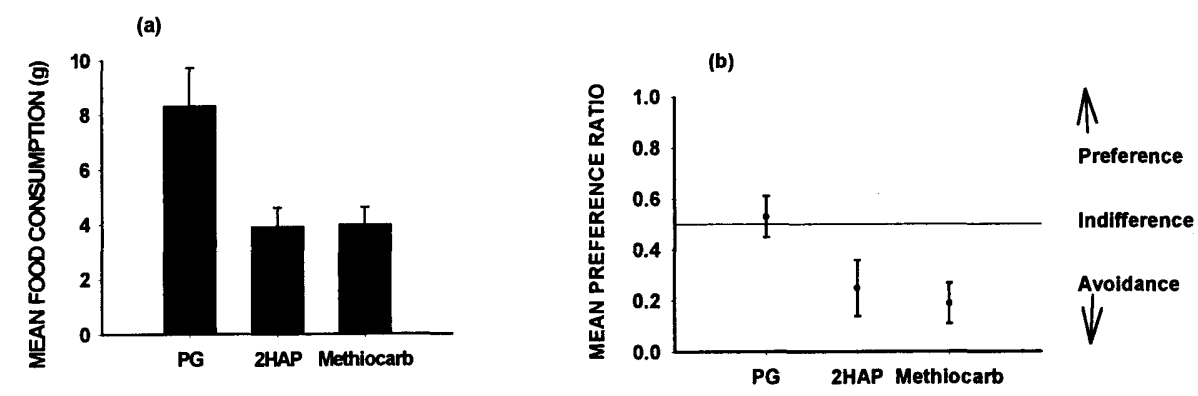

Figure 2a. Mean ( $\pm S E$ ) consumption ( $g$ ) of food during training. Figure 2b. Mean preference ratios ( $\pm S E$ ) of starlings during 2 choice test with standard cage door.

\section{Experiment 3}

The objectives of Experiment 3 were to (1) evaluate the effect of lengthening the duration of habituation to the plexiglass cage door, and (2) compare the behavioral mechanisms of starlings to primary and secondary repellents when the peripheral senses are not bypassed.

\section{Food Intake During Training}

Food consumption was not affected by the combined effects of repellents (Table II). Starlings that fed on chow dosed with 2HAP $(P=0.18)$ and MA $(P=$ $0.11)$ slightly reduced food consumption compared to controls (Figure $3 a$ ). However, starlings with methiocarb in their feed exhibited more of a reduced food intake $(P=$ 0.026).

\section{2-Choice Learning Test}

Application of repellents on food affected the subsequent 2-choice preference ratios of the starlings $\left(\mathrm{F}_{3,20}=6.09, P=0.004\right)$. Starlings that were 


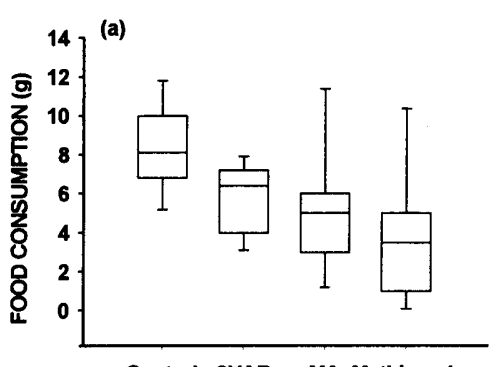

Control 2HAP MA Methiocarb

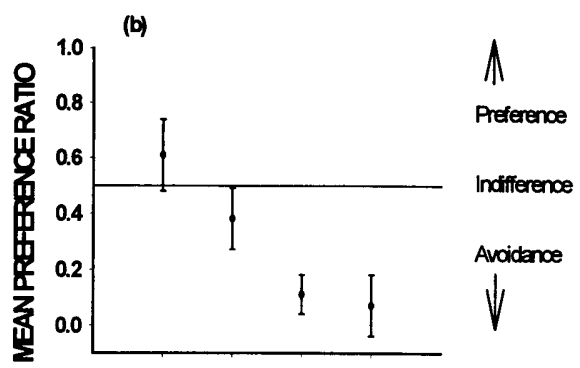

Control 2HAP MA Metriocart

Figure 3a. Mean ( $\pm S E$ ) consumption (g) of food by starlings during 2 hour exposure to topically applied repellents. The upper and lower capped vertical bars represent $95^{\text {th }}$ and $5^{\text {th }}$ percentiles and the upper and lower ends of the boxes represent the $75^{\text {th }}$ and $25^{\text {th }}$ percentiles, respectively. The horizontal mark through the box represents the median. Figure $3 b$. Mean preference ratios $( \pm S E$ ) of starlings during 2-choice test after extended adaptation to plexiglass cage door (60 hours).

provided food treated with $2 \mathrm{HAP}$ did not develop an aversion to the colored food cup $(P=0.123)$ (Figure $3 \mathrm{~b})$. However, starlings fed chow treated with MA $(P=0.002)$ and methiocarb $(P=0.001)$ ate less from the colored food cup, indicating strong food aversion learning.

\section{Behavioral Analyses}

Compared to controls, starlings fed pellets treated with methiocarb tended to reduce the proportion of time feeding $(P=0.023)$ and decrease the duration of feeding bouts $(P=0.101)$ (Figure 4a, 4b). Starlings that ate pellets treated with the primary repellents, $2 \mathrm{HAP}$ and MA, did not reduce their total feeding time $(P=0.97)$ or duration of feeding bouts $(P=0.709)$ in comparison to controls. Comparison of primary repellents and the secondary repellent, methiocarb, indicates that the methiocarb starlings devoted less of their activity budget to feeding $(P=0.014)$, and demonstrated a slight reduction in the duration of feeding bouts $(P=0.132)$.

We measured the feeding efficiency of starlings by quantifying the number of pellets consumed per minute of feeding (Figure 5a). Starlings that were provided food with the primary repellents showed a tendency to reduce efficiency compared to both controls $(P=0.129)$ and starlings with methiocarb in their feed $(P=0.140)$.

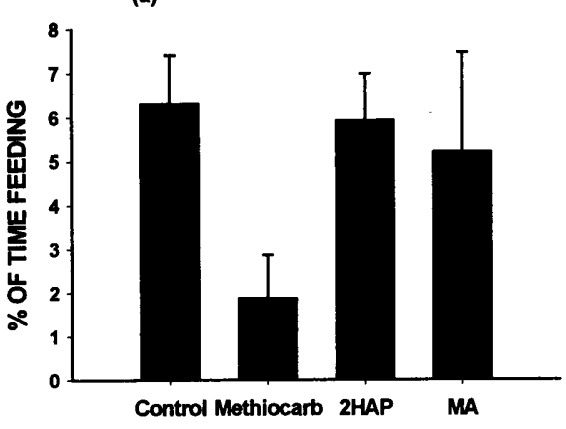

(b)

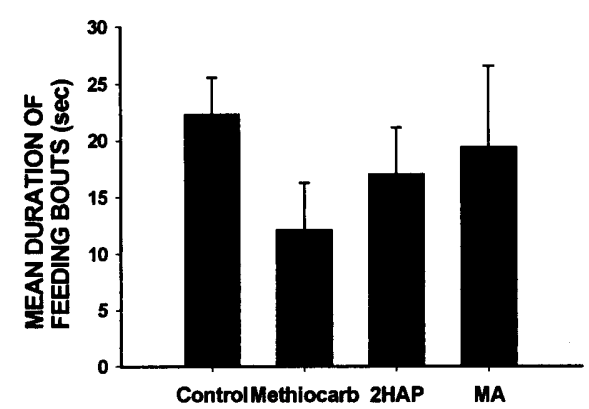

Figure $4 a$. Mean percent time $( \pm S E)$ starlings were engaged in feeding bouts during 2 hour training period. Figure $4 b$. Mean duration ( $\pm S E$ ) of feeding bouts of starlings during 2 hour training period.

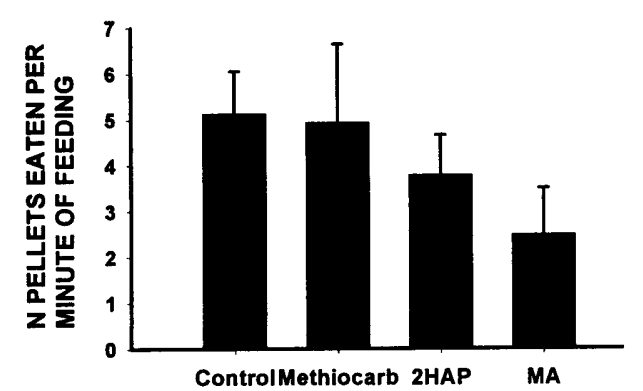

(b)

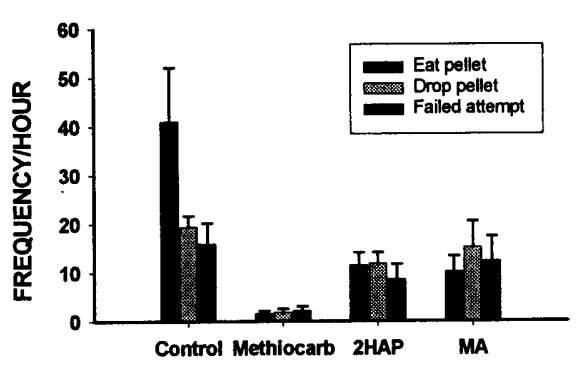

Figure 5a. Mean feeding efficiency (\# of pellets consumed/minute of feeding) ( $\pm S E$ ) of starlings during 2 hour training period. Figure $5 b$. Mean frequency $( \pm S E)$ of food pellets eaten, food pellets dropped, and failed attempts (bird searches in food cup but does not pick up any pellets) of starlings during 2 hour training period. 
Feeding efficiency of starlings treated with methiocarb was not different from controls $(P=0.679)$ (Figure 5a), although the latter group spent less total time feeding (Figure $4 \mathrm{a})$. Controls ate more frequently than those fed primary repellents $(P=0.018)$ or methiocarb $(P=0.004)$. Starlings fed primary repellents ate slightly more frequently than birds with methiocarb $(P=0.18)$. The frequency of dropped pellets and failed attempts among controls was not different from the primary

repellents $(P=0.56)$, but controls demonstrated these behaviors more frequently than those fed methiocarb $(P=0.048)$. Starlings fed primary repellents dropped and failed to secure food more frequently than with methiocarb $(P=0.037)$ (Figure 5b).

To evaluate behavioral mechanisms we measured feeding frequency and number of pellets eaten at 30 minute intervals. The control and primary repellent groups ate most frequently at the $0-30$ minute and $30-60$ minute time periods, followed by a slight decrease at the $60-90$ minute and $90-120$ minute intervals (Figure 6). The methiocarb group exhibited several differences: these starlings decreased food consumption rates and frequency of feeding (Figure 6).

Evaluation of the number of pellets eaten per 30 minute interval demonstrates the operation of different mechanisms in response to the different treatments (Figure 7). Control starlings increased the number of pellets eaten during the $60-90$ minute and $90-120$ minute intervals, indicating a possible compensation for the reduced frequency of feeding bouts as observed in Figure 6 . Starlings with MA in their feed ate about the same number of repellents as controls during the first 30 minutes, but then exhibited a sharp decline in consumption rates. Finally, starlings with $2 \mathrm{HAP}$ in their food exhibited consistent consumption rates throughout each 30 minute interval. Among the starlings treated with methiocarb, pellet consumption was minimal: $94 \%(n=17)$ of pellets eaten were consumed within the first 30 minutes; and no pellets were eaten after 60 minutes.

Compared to controls, starlings fed repellent-treated foods reduced the frequency of drinks $(P<0.01)$ (Figure 8 ). The frequency of drinks for the 30 minute time intervals was consistent among controls (Figure 8). Starlings with methiocarb reduced the frequency of drinks after the first 30 minutes (Figure 8). Although starlings fed foods treated with the primary repellents also drank less frequently than the controls, these starlings appeared to exhibit a different pattern than methiocarb (Fig 8). The MA and 2HAP starlings drank less frequently during the first 30 minutes, then tended to increase drinking in the $30-60$ minute interval.

In addition to measuring feeding behavior in response to the repellents, we evaluated evidence of gastrointestinal illness (i.e., the unconditional response) by quantifying the frequency of regurgitation. Control starlings ( 2 of 6 ) visibly regurgitated their food on 3 occasions during the 2 hours of observation, but no pattern was apparent (Figure 9). A majority of starlings with MA (4 of 6) in their feed regurgitated during the 2 hour observation period. Regurgitation occurred most frequently during the first 30 minutes $(x=1.5 \pm 0.9 \mathrm{SE})$. Only 2 of 6 starlings with 2HAP in their feed regurgitated during the 2 hours, and like controls no pattern was

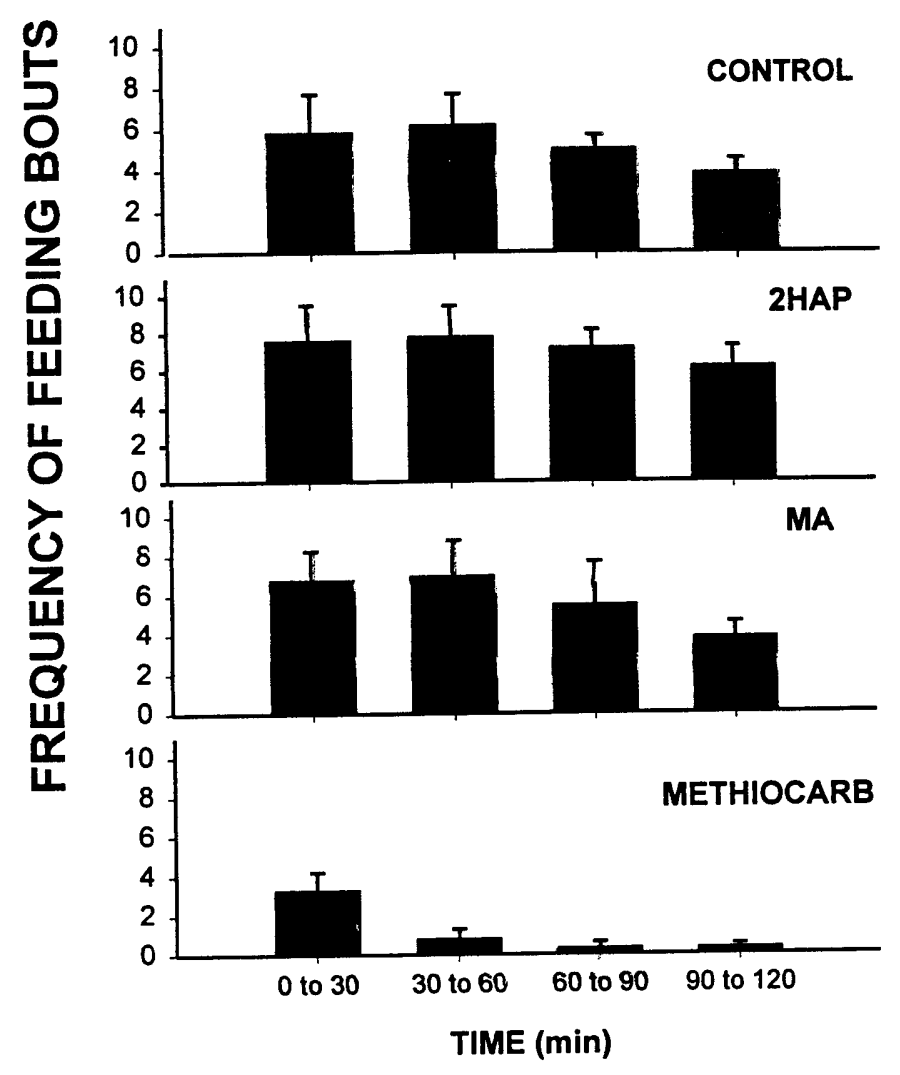

Figure 6. Mean frequency $( \pm S E)$ of feeding bouts during 30 min intervals. 


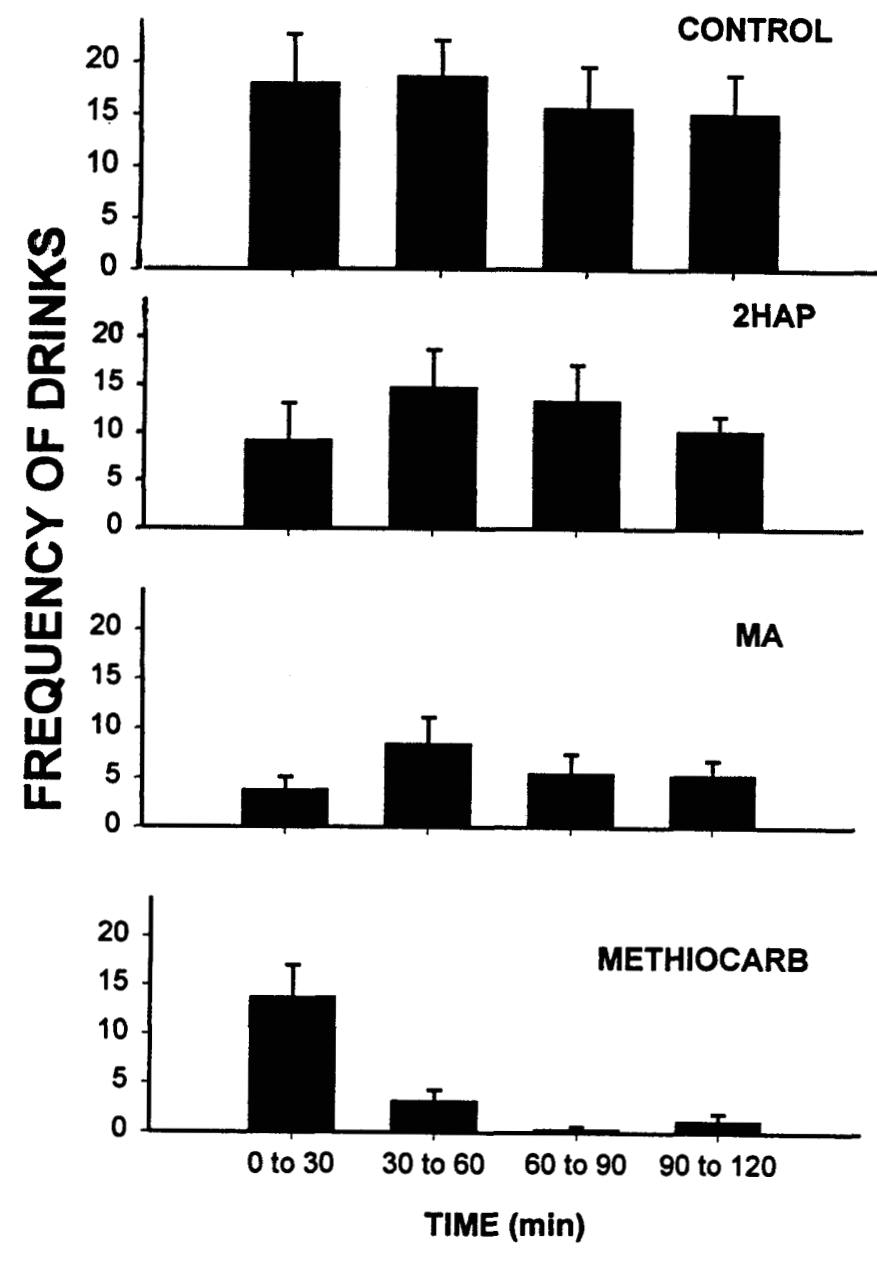

Figure 7. Mean frequency $( \pm S E$ ) of food pellets eaten during 30 min intervals.

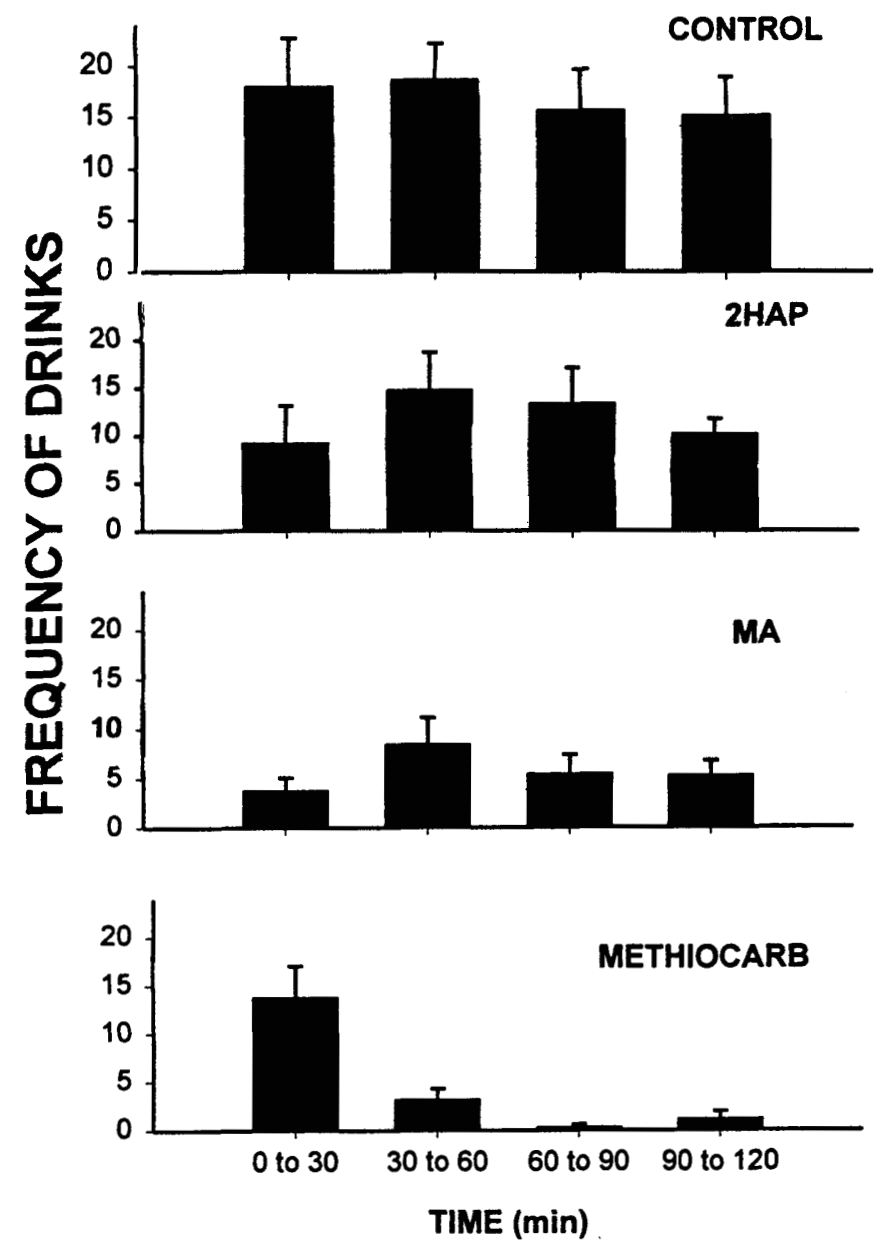

Figure 8. Mean frequency ( $\pm S E$ ) of drinks during 30 min intervals. 


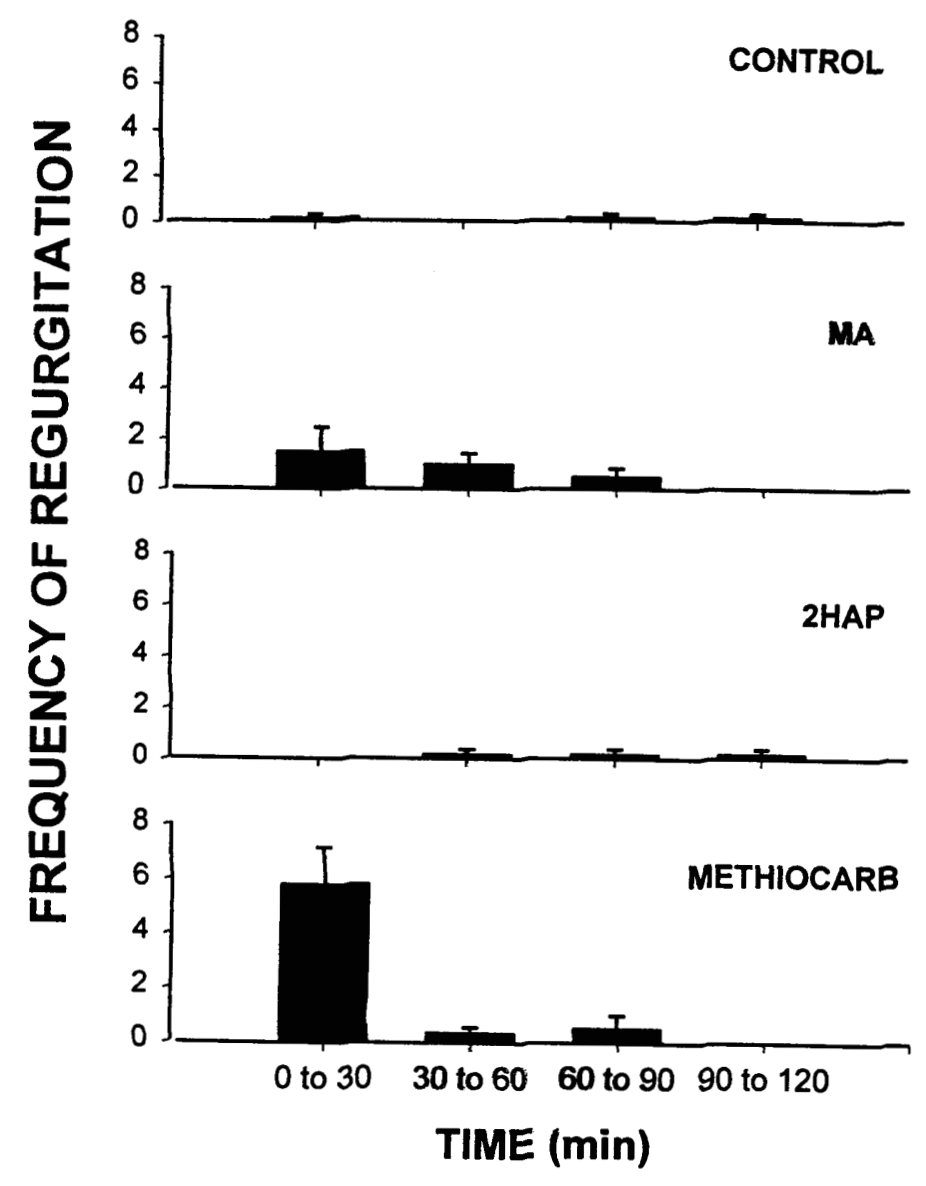

Figure 9. Mean frequency ( $\pm S E$ ) of regurgitation during 30 min intervals of 2 hour training period. apparent. As expected, evidence of gastrointestinal illness was observed most frequently among the starlings treated with methiocarb, where 6 of 6 regurgitated. Mean regurgitation during the first 30 minutes was $5.8 \pm 1.9 \mathrm{SE}$ (Figure 9).

\section{Discussion}

Results from these experiments, and from a similar set of studies (Sayre and Clark in review), indicate that the efficacy of primary repellents can be enhanced via gastrointestinal delivery. Topical application of 2HAP in Experiment 3 showed that this repellent was completely ineffective as an aversive conditioner. However, when the peripheral senses were by-passed in Experiment 2, the starlings displayed a tendency to avoid food they associated with the visual cue. When MA was compared with methiocarb in a parallel experiment (Sayre and Clark in review), this primary repellent was as effective as methiocarb.

Starlings in Experiment 1 did not develop food aversion learning from either primary or secondary repellents. This was unexpected because methiocarb has been demonstrated to effectively condition birds to avoid foods ${ }^{13,14,17}$. We hypothesized that the anomalous results were due to concurrent interference ${ }^{20,21}$. Specifically, that replacement of the standard cage door with a clear plexiglass door 16 hours prior to training distracted the starlings from learning to associate the colored food cup with the illness caused by the repellent. Results from Experiments 2 and 3 supported our hypothesis that alteration of the visual environment mitigated aversive conditioning. Starlings exposed to methiocarb developed aversions to the colored food cup when their cage door was not replaced (Experiment 2), and when they habituated to the plexiglass cage door (Experiment 3 ).

Experiment 3 compared the effect of topical (i.e., unmasked) application of primary repellents $2 \mathrm{HAP}$ and methyl anthranilate, and methiocarb a secondary repellent. The amount of food consumed during training did not decrease with the primary repellents, but the starlings did reduce consumption of foods treated with methiocarb.

The behavioral data demonstrate different behavioral mechanisms in response to the 3 repellents. Methiocarb, the secondary repellent, is an emetic and a cholinesterase inhibitor. The starlings in the methiocarb group initially ate a few pellets, but quickly became ill. Thereafter these starlings exhibited a marked reduction in feeding and drinking during the 2 hour training period. Although starlings that were fed methiocarb demonstrated a relatively high feeding efficiency per minute of feeding, the overall proportion of time feeding was reduced.

As expected, the behavioral response to primary repellents, $2 \mathrm{HAP}$ and MA, were different than controls or to methiocarb. In comparison to controls, starlings 
that ate food treated with 2HAP and MA showed only a very slight reduction in proportion of time feeding or duration of feeding bouts. However, the foraging efficiency decreased compared to controls. Compared to controls, starlings fed primary repellents also had a greater ratio of drops and failed attempts in relation to pellets consumed. These data demonstrate that $2 \mathrm{HAP}$ and MA act as irritants.

There were also some interesting behavioral differences between responses to the 2 primary repellents. Comparison between 30 minute time blocks indicate that starlings with MA in their diet reduced the frequency of feeding bouts and the number of pellets eaten from the $0-30$ minute to $90-120$ minute time blocks. These data suggest that MA also might act as a secondary repellent. On the other hand, starlings with $2 \mathrm{HAP}$ in their food did not reduce the frequency of feeding bouts or number of pellets eaten as time progressed in the training period. These data demonstrate that 2HAP irritates the peripheral senses but birds continue to sample the food.

These subtle behavioral differences help explain the results from the 2choice learning trials. As expected, adding the secondary repellent, methiocarb, into food resulted in markedly reduced food consumption and gastrointestinal illness during training. The starlings demonstrated food aversion learning during the 2choice test. MA appears to have acted as both a primary and a secondary repellent. Compared to starlings with methiocarb in their food, those provided foods treated with MA continued feeding, although their efficiency decreased. Several of these starlings (4 of 6) developed gastrointestinal illness, although the illness did not appear to be as intense as with methiocarb. Starlings with MA in their feed subsequently demonstrated a strong aversion to the colored food cup. Trigeminal irritants such a methyl anthranilate do not result in conditioned odor avoidance in birds ${ }^{21}$. Evidence suggests that the although odor is a good potentiator of avoidance learning, it is not as strong as taste at inducing conditioned avoidance of foods ${ }^{22,23,24,25,26}$. In contrast, gustatory and chemesthetic cues (which irritate peripheral as well as gastrointestinal nerves) will result in food avoidance learning.

Treatment of food with the primary repellent $2 \mathrm{HAP}$ resulted in reduced feeding efficiency, but the test starlings did not develop gastrointestinal illness. Moreover, as time progressed the frequency of feeding bouts, and the number of pellets eaten per bout did not change. As expected with a primary repellent, 2HAP irritated the peripheral senses, but did not induce illness. Thus, the starlings did not develop learned aversion.

The reduction in drinking among all groups with repellent in their food was unexpected. The avoidance of drinking could be explained by a location effect ${ }^{17}$, whereby starlings avoided a proximally located food cup and drink tube. It is also possible that application of repellent from food simply reduces all consumptive behavior, perhaps due to irritation of nerves in the oral and nasal cavity.

\section{Management Implications}

The challenge ahead is to mask the irritating qualities of primary repellents so birds will ingest sufficient quantities to incur gastrointestinal illness and subsequent food aversion learning. This can be achieved by micro-encapsulating repellents so that the aversive properties are masked to a bird's peripheral senses, but are released enterically. Conversion of primary repellents to secondary repellents holds promise, both for a new way of conceptualizing the formulations of primary repellents but also for paving the way to develop new repellent formulation that can rely on compounds that are less toxic or environmentally harmful. The development of primary repellents will focus on naturally occurring products that are considered toxicologically and environmentally safe. Some candidates, such as MA, are food additives that have been approved for human consumption. MA has been demonstrated to be safe, even when consumed in high doses. The formulations used for bird repellents will not be at toxic concentrations. Using chemicals such as these would provide wildlife managers and agriculturists with a tool that is effective, environmentally and safe.

The sensitivity of birds in these experiments to visual stimuli (i.e., interference due to the plexiglass cage door) demonstrates the critical importance involved with the presentation and timing of cues. Signals from the sender (the wildlife manager) may not be interpreted by the receivers (the animals) in the way that was intended. A successful operation to develop conditioned food avoidance learning requires careful planning to ensure that birds learn to associate a visual cue with exposure to the repellents. Otherwise, the effects of concurrent interference can counteract even the most effective repellents.

\section{References}

1. Rogers, J. G., Jr. Journal of Wildlife Management 1974, 38, 418-423.

2. Dolbeer, R. A.; Avery, M. L; Tobin, M. E. Pesticide Science 1994, 40, 147 161.

3. Fagerstone, K. A.; Shafer, E. W. Jr. Proceedings of the Vertebrate Pest Conference 1998, 18, 319-324.

4. Clark, L. Current Ornithology 1998, 14, 1-37.

5. The principles of learning and behavior; Domjan, M.; Brooks/Cole: Pacific Grove, CA, USA, 1998.

6. Conover, M. R. Journal of Applied Ecology 1984, 21, 437-443.

7. Glahn, J. F.; Mason, J. R.; Woods, D. R. Wildlife Society Bulletin 1989, 17, 313-320.

8. Mason, J. R.; Glahn, J. F.; Reidinger, R. F.; Dolbeer, R. A. Journal of Wildlife Management 1985, 49, 636-642.

9. Mason, J. R.; Clark, L. Proceedings of the Vertebrate Pest Conference $1992,15,115-129$. 
10. Fenaroli's Handbook of Flavor Ingredients. 1971. Furia, T. E. and N. Bellanca, Editors. The Chemical Rubber $\mathrm{CO}, \mathrm{Cleveland,} \mathrm{OH}$.

11. Clark, L.; Shah, P. S. Journal of Chemical Ecology 1994, 20, 321-339.

12. Shah, P. S.; Mason, J. R.; Clark, L. Chemical Signals in Vertebrates, VI, Doty, R. L.; Muller-Schwarze, D., editors, Plenum Press. New York, USA, 1992, 291-296.

13. Mason, J. R.; Shah, P. S.; Clark, L. Journal of Wildlife Management 1991, 55, 334-340.

14. Avery, M. L. Journal of Wildlife Management 1985, 49, 1116-1121.

15. Mason, J. R.; Reidinger, R. F. Journal of Wildlife Management 1983, 47, 383-393.

16. Mason J. R. Social Learning: physiological and biological perspectives, Zentall, T.R.; Galef, B. G., editors, Lawrence Erlbaum Associates, Publishers, Hilldale, New Jersey, USA, 1987, 99-115.

17. Mason J. R; Reidinger, R.F. The Auk 1982, 99, 548-554.

18. Tobin, M. E. Journal of Wildlife Management 1985, 49, 1102-1108.

19. Mason, J. R.; Arzt, A. H.; Reidinger, R. F. The Auk 1984, 101, 796-803.

20. Kamin, L. J. Punishment and Aversive Behavior, Campbell, B. A.; Church, R.M., editors. Appleton-Century-Crofts. New York, USA, 1969.

21. Revusky, S. Food Aversion Learning, Milgram, N. W.; Krames, L.; Alloway, T.M., editors, Plenum Press. New York, USA, 1977, 1-51.

22. Clark, L. Wilson Bulletin 1996, 108, 36-52.

23. Hargrave, G. E.; Bolles R. C. Psyconomic Science 1971, 23, 91-92.

24. Launchbaugh, K. L.; Provenza, F. D.; Burritt, E. A. Journal of Chemical Ecology 1993, 19, 1047-1056.

25. Mason J. R.; Adams, M. A.; Clark, L. Journal of Wildlife Management 1989, 53, 55-64.

26. Nachman, M.; Rauschenberger, J.; Ashe, J. H. Food Aversion Learning, Milgram, N. W.; Krames, L.; Alloway, T. M., editors, Plenum Press. New York, USA, 1977, 105-131. 